Conclusion Retinal vascular disease may be frequent in patients with RA. The presence of aPL may be associated with a higher prevalence of retinal abnormalities in RA patients.

\section{THU0145 HYPERHOMOCYSTEINEMIA IN RHEUMATOID ARTHRITIS}

B Seriolo, V Ferretti, D Fasciolo, M Cutolo. Division of Rheumatology, Department of Internal Medicine and Medical Specialities, University of Genova, Genova, Italy

\subsection{6/annrheumdis-2001.1047}

Background Recent reports have suggested that elevated total circulating homocysteine levels are important risk factor for vascular diseases, stroke, and thrombotic events in patients with atherosclerosis and in patients with systemic lupus erythematosus.

Objectives The aim of our study was to analyse the relationship between plasma homocysteine levels and thrombotic events in a select population of rheumatoid arthritis (RA) patients with or without antiphospholipid antibody (aPL) positivity.

Methods 168 female RA patients attending the Extra-articular Involvement RA Clinic of University of Genova and 72 female subjects matched for age and vascular diseases as controls were included in the study. 30 of the RA patients showed aPL antibody positivity and 138 were found aPL antibody negativity on the basis of the concomitant presence or absence of high concentrations of anticardiolipin (aCL) antibodies or presence of Lupus Anticoagulant (LA). All subjects were evaluated for plasma homocysteine concentrations and for the occurrence of thrombotic events.

Results Twenty-five RA patients and five controls reported a history of thrombotic events. Eleven and nine of RA patients with aPL antibody positivity were found previously affected by venous and arterial thrombosis, respectively. Plasma levels of homocysteine in aPL antibody positive patients with thrombosis were found significantly higher than in aPL antibody positive RA patients without thrombosis $(\mathrm{p}<0.001)$. When RA patients with thrombosis were analysed a significant increase of plasma homocysteine levels was found in aPL antibody positive RA patients versus aPL antibody negative RA patients $(p<0.04)$ and versus related controls $(\mathrm{p}<0.003)$.

Conclusion The association observed between aPL antibody positivity and high levels of plasma homocysteine in RA patients may represent a possible risk factor for thrombotic events. Therefore, it is suggested that hyperhomocysteinemia might be involved in vascular-related mortality observed in RA patients with a history of thrombosis.

\section{THU0146 REAL-WORLD EXPERIENCE WITH INFLIXIMAB IN THE TREATMENT OF RHEUMATOID ARTHRITIS: A SUBGROUP ANALYSIS OF METHOTREXATE RECEIVING AND NON- RECEIVING PATIENTS}

D Erkan, Y Yazici, I Kulman, K Tai, J Markenson, MJ Harrison. Division of Rheumatology, Hospital for Special Surgery, Weill Medical College of Cornell University, New York, USA

\subsection{6/annrheumdis-2001.1048}

Background Infliximab is a chimeric monoclonal anti-TNF antibody developed to treat rheumatoid arthritis (RA) patients who have had a less than optimal response to previous therapy. It has been recommended that infliximab be given concomitantly with methotrexate (MTX) to prevent the development of autoantibodies.

Objectives To identify clinical response and adverse events in RA patients treated with infliximab for a period of 6 months, with a subgroup analysis of MTX-receiving (MTX-R) and non-receiving (MTX-NR) patients.

Methods RA patients receiving infliximab at our ambulatory infusion unit completed questionnaires regarding RA history, treatment, and measures of disease activity (HAQ) at baseline, and at 2 weeks, 8 weeks, and every 2 months thereafter. Patients were queried about side effects experienced between infusions. ANOVA statistics were used for comparison.

Results 36 patients were started on infliximab between JanuaryJuly 2000 (F/M: 33/3, mean age: $63+12.9$ y, mean disease duration: $15.3+10.2$ y) and 24 patients completed 6 months of treatment. Patients had failed an average of 3 DMARDs prior to infliximab. HAQ scores for $24 / 36$ patients who completed 6 months of treatment significantly improved from $1.95+0.60$ to $1.21+0.67$ ( $p=0.0002)$. In subgroup analysis, the baseline characteristics of MTX-R and MTX-NR groups were similar with respect to age and disease duration. Twenty (56\%) patients were not receiving MTX due to history of adverse event, allergic reaction or patient refusal. For patients who completed 6 months of infliximab: in MTX-R group, HAQ scores for 14/24 patients improved from $1.99+0.58$ to $1.34+0.54(\mathrm{p}=0.005)$; and in MTX-NR group HAQ scores for 10/24 patients improved from $1.89+0.65$ to $1.05+0.81(\mathrm{p}=0.02)$. During 6 months of follow-up, 3/10 (30\%) of MTX-NR and 9/14 (64\%) of MTX-R patients had a total of 23 adverse events after 198 infusions. There was no difference between the 2 subgroups with respect to the type of adverse events. Ten MTX-NR patients discontinued treatment before 6 months (adverse events: 7, inadequate response to treatment: 2 , and non-compliance: 1 . Only 2 MTX-R patients discontinued treatment prior to 6 months, due to inadequate response.

Conclusion Following 6 months of infliximab treatment, RA patients with long-standing disease demonstrate a clinically important and statistically significant improvement in functional disability as measured by HAQ. However, our subgroup analysis suggests that infliximab administered without MTX carries a higher rate of discontinuation. These findings may be due to patient characteristics or our small sample size. Continuing follow-up of patients receiving infliximab in the absence of MTX will provide a better understanding of the true risk/benefit of infliximab used alone.

\section{THU0147 TRACKING RA EROSIONS OVER THE FIRST 2 YEARS OF DISEASE: AN MR AND XR STUDY}

FM McQueen, N Benton, J Crabbe, E Robinson, S Yeoman, L McLean, N Stewart. Departments of Rheumatology and Radiology, Auckland Hospital, Auckland, New Zealand

10.1136/annrheumdis-2001.1049

Background MRI is a highly sensitive technique for revealing rheumatoid erosions. Studies from this cohort have revealed MR wrist erosions in $45 \%$ and $\mathrm{XR}$ erosions in $12 \%$ of early $\mathrm{RA}$ patients. ${ }^{1}$ It has been proposed that MR erosions progress to XR erosions during the course of RA but lesion-based evidence for this is scarce. We have used MR and XR data from this cohort to examine the predictive value of a baseline $\mathrm{MR}$ score and to track the fate of individual lesions over a 2 year period.

Objectives 